\title{
PNPLA3 is the dominant SNP linked to liver disease severity at time of first referral to a tertiary center
}

Balcar, Lorenz ; Semmler, Georg ; Oberkofler, Hannes ; Zandanell, Stephan ; Strasser, Michael ; Datz, Leonora ; Niederseer, David ; Feldman, Alexandra ; Stickel, Felix ; Datz, Christian ; Paulweber,

\author{
Bernhard ; Aigner, Elmar
}

\begin{abstract}
BACKGROUND Single nucleotide polymorphisms (SNPs) in genes including PNPLA3, TM6SF2, HSD17B13 and SERPINA1 have been identified as risk modifiers of progression in chronic liver disease (CLD). However, it is unclear whether genotyping for these risk variants is useful in clinical routine. METHODS Liver disease severity was assessed by liver stiffness measurement (LSM) and by presence of clinical manifestations of advanced-chronic liver disease (ACLD) in 779 consecutive CLD patients at the time of referral to a tertiary center. The associations of risk variants with CLD severity were calculated individually and in a combined model using a polygenic risk-score. RESULTS Non-alcoholic fatty liver disease (NAFLD) was the most common etiology $(\mathrm{n}=511,65.6 \%)$, and ACLD was present in $217(27.9 \%)$ patients. The PNPLA3-G-allele remained independently associated with higher LSM (adjusted-B: 2.508 [95\%CI: 0.887-4.130], $\mathrm{P}=0.002$ ) or the presence of ACLD (aOR: 1.562 [95\%CI: 1.097-2.226], $\mathrm{P}=0.013$ ). SERPINA1-Z-allele was also independently associated with LSM (adjusted-B: 4.558 [95\%CI: 1.182-7.934], $\mathrm{P}=0.008$ ), while the other risk alleles did not attain statistical significance. Combining these risk alleles into a polygenic risk-score was significantly associated with LSM (adjusted-B: 0.948 [95\%CI: 0.153-1.743], $\mathrm{P}=0.020$ ). CONCLUSION PNPLA3 risk-variants are linked to liver disease severity at the time of first referral to an outpatient hepatology clinic.
\end{abstract}

DOI: https://doi.org/10.1016/j.dld.2021.06.015

Posted at the Zurich Open Repository and Archive, University of Zurich

ZORA URL: https://doi.org/10.5167/uzh-215314

Journal Article

Published Version

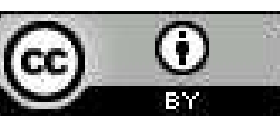

The following work is licensed under a Creative Commons: Attribution 4.0 International (CC BY 4.0) License.

Originally published at:

Balcar, Lorenz; Semmler, Georg; Oberkofler, Hannes; Zandanell, Stephan; Strasser, Michael; Datz, Leonora; Niederseer, David; Feldman, Alexandra; Stickel, Felix; Datz, Christian; Paulweber, Bernhard; Aigner, Elmar (2022). PNPLA3 is the dominant SNP linked to liver disease severity at time of first referral to a tertiary center. Digestive and Liver Disease, 54(1):84-90.

DOI: https://doi.org/10.1016/j.dld.2021.06.015 
Liver, Pancreas and Biliary Tract

\title{
PNPLA3 is the dominant SNP linked to liver disease severity at time of first referral to a tertiary center
}

\author{
Lorenz Balcar ${ }^{\mathrm{a}, \mathrm{b}, 1}$, Georg Semmler ${ }^{\mathrm{a}, \mathrm{b}, \mathrm{c}, 1}$, Hannes Oberkofler ${ }^{\mathrm{a}}$, Stephan Zandanell ${ }^{\mathrm{a}}$, \\ Michael Strasser ${ }^{\mathrm{a}}$, Leonora Datz ${ }^{\mathrm{c}}$, David Niederseer ${ }^{\mathrm{d}}$, Alexandra Feldman ${ }^{\mathrm{a}}$, Felix Stickel ${ }^{\mathrm{e}}$, \\ Christian Datz ${ }^{c}$, Bernhard Paulweber ${ }^{\mathrm{a}}$, Elmar Aigner ${ }^{\mathrm{a}, *}$ \\ a First Department of Medicine, Paracelsus Medical University Salzburg, Salzburg, Austria \\ ${ }^{\mathrm{b}}$ Division of Gastroenterology and Hepatology, Department of Internal Medicine III, Medical University of Vienna, Vienna, Austria \\ ' Department of Internal Medicine, General Hospital Oberndorf, Teaching Hospital of the Paracelsus Medical University Salzburg, Salzburg, Austria \\ ${ }^{\mathrm{d}}$ Department of Cardiology, University Hospital Zurich, Zurich, Switzerland \\ e Department of Gastroenterology and Hepatology, University Hospital Zurich, Zurich, Switzerland
}

\section{A R T I C L E I N F O}

\section{Article history:}

Received 12 April 2021

Revised 26 May 2021

Accepted 14 June 2021

Available online 11 July 2021

\section{Keywords:}

Cirrhosis

Genetic risk

HSD17B13

PNPLA3

SERPINA1

TM6SF2

\begin{abstract}
A B S T R A C T
Background: Single nucleotide polymorphisms (SNPs) in genes including PNPLA3, TM6SF2, HSD17B13 and SERPINA1 have been identified as risk modifiers of progression in chronic liver disease (CLD). However, it is unclear whether genotyping for these risk variants is useful in clinical routine.

Methods: Liver disease severity was assessed by liver stiffness measurement (LSM) and by presence of clinical manifestations of advanced-chronic liver disease (ACLD) in 779 consecutive CLD patients at the time of referral to a tertiary center. The associations of risk variants with CLD severity were calculated individually and in a combined model using a polygenic risk-score.

Results: Non-alcoholic fatty liver disease (NAFLD) was the most common etiology $(n=511,65.6 \%$, and ACLD was present in 217 (27.9\%) patients. The PNPLA3-G-allele remained independently associated with higher LSM (adjusted-B: 2.508 [95\%CI: $0.887-4.130], P=0.002$ ) or the presence of ACLD (aOR: 1.562 [95\%CI: 1.097-2.226], $P=0.013$ ). SERPINA1-Z-allele was also independently associated with LSM (adjusted-B: 4.558 [95\%CI: 1.182-7.934], $P=0.008$ ), while the other risk alleles did not attain statistical significance. Combining these risk alleles into a polygenic risk-score was significantly associated with LSM (adjusted-B: 0.948 [95\%CI: 0.153-1.743], $P=0.020$ ).
\end{abstract}

Conclusion: PNPLA3 risk-variants are linked to liver disease severity at the time of first referral to an outpatient hepatology clinic.

(C) 2021 The Author(s). Published by Elsevier Ltd on behalf of Editrice Gastroenterologica Italiana S.r.l. This is an open access article under the CC BY license (http://creativecommons.org/licenses/by/4.0/)

\section{Introduction}

Several single nucleotide polymorphisms (SNPs) have been identified and confirmed to modify the risk of progression in chronic liver disease (CLD) of different etiologies. In the first place, the rs738409 $\mathrm{G}$-allele encoding the $I 148 \mathrm{M}$ variant of patatinlike phospholipase domain-containing protein 3 (PNPLA3) was linked with non-alcoholic fatty liver disease (NAFLD) and non-alcoholic steatohepatitis (NASH) in the general population [1,2]. Moreover, it has been shown to increase the progression to cirrhosis in

\footnotetext{
* Corresponding author.

E-mail address: e.aigner@salk.at (E. Aigner).

1 Lorenz Balcar and Georg Semmler contributed equally.
}

alcoholic liver disease (ALD)- [3-6] and alcoholic steatohepatitis (ASH) [7], but also in patients with chronic hepatitis C [8], and to augment the risk for developing hepatocellular carcinoma (HCC) $[9,10]$. Further genome-wide association studies have linked the transmembrane 6 superfamily 2 (TM6SF2) rs58542926 variant to NAFLD severity [11], and the rs72613567 TA-allele of the $17 \beta$ hydroxysteroid dehydrogenase type 13 (HSD17B13) gene to a reduced risks of ALD, chronic hepatitis C, NAFLD and NAFLD-associated cirrhosis [12,13]. Finally, the SERPINA1 p.E342K allele has recently been associated with disease severity and an increased risk of developing cirrhosis in patients with NAFLD and ALD [14]. Although predominantly studied in NAFLD/NASH, evidence from ALD and viral hepatitis highlight the relevance of these SNPs for disease progression across CLD etiologies, with the variant allele being more 
prevalent (PNPLA3, SERPINA1) and less prevalent (HSD17B13) in advanced stages [14-18]. Therefore, we aimed at investigating the usefulness of genotyping for four important SNPs (PNPLA3, TM6SF2, HSD17B13, SERPINA1) in clinical routine for the assessment of the severity of CLD.

\section{Methods}

\subsection{Patients and definitions}

All patients who attended the hepatic outpatient clinic of the Paracelsus Medical University Salzburg for the first time, between $10 / 2018$ and $07 / 2020$, were evaluated for inclusion in this retrospective cross-sectional analysis. Analysis of the genetic risk variants was commenced as part of the clinical routine in 10/2018 as part of the laboratory evaluation of all patients at first time assessment and data from these 875 subjects were collected for analysis. Patients were only included if information on all SNPs ( $P N-$ PLA3, TM6SF2, HSD17B13, SERPINA1) was available. Patients were excluded, if any of the following criteria were present: Patients with a history of orthotopic liver transplantation $(n=4)$, liver metastasis of other origin than HCC $(n=4)$, cholestatic or autoimmune liver disease (i.e., autoimmune hepatitis [AIH, $n=18$ ], primary biliary or sclerosing cholangitis [PBC, $n=18 / \mathrm{PSC}, n=1$ ]), conditions causing temporarily elevated transaminases in the absence of CLD ( $n=9$ ), drug-induced liver disease (DILI, $n=3$ ) or Wilson disease $(n=2)$, both due to the lack of data of risk variants in these diseases and/or the low number of patients with these etiologies. Finally, all patients with invalid liver stiffness measurement (LSM) were also excluded ( $n=37)$. Data were obtained by retrospective evaluation of medical records.

The amount of alcohol consumed was estimated as number of drinks per day/week and significant alcohol abuse was defined as $\geq 3$ drinks per day for men and $\geq 2$ drinks for women [19]. This threshold was used for allocation of a fatty liver to alcoholic origin in this analysis, also if components of metabolic syndrome as indicators of NAFLD were present. Hepatic decompensation was defined as current or previous presence of ascites, hepatic encephalopathy (HE), or variceal bleeding. ACLD was defined as $\mathrm{LSM} \geq 10 \mathrm{kPa}$, unequivocal clinical signs of cirrhosis or history of hepatic decompensation [20]. Also, LSM was stratified according to the BAVENO-VI consensus recommendations for "suspected ACLD" ( $\geq 10 \mathrm{kPa}$ ), "highly suggestive ACLD" ( $\geq 15 \mathrm{kPa}$ ), and ruling-in clinically significant portal hypertension (CSPH; $\geq 20-25 \mathrm{kPa}$ ) [21]. This study was approved by the ethics committee of Salzburg and performed in accordance with the standards of the Declaration of Helsinki. Since it was a retrospective analysis of available clinical data, the requirement of a written informed consent was waived.

\subsection{LSM and controlled attenuation parameter measurement}

LSM and controlled attenuation parameter (CAP) measurements were performed by transient elastography using a FibroScan ${ }^{\circledR}$ (Echosens, Paris, France) applying previously published reliability criteria [22]. Measurements were carried out after a fasting period of at least $3 \mathrm{~h}$. The $\mathrm{M}$ and XL-probe were chosen based on the expertise of the manufacturer. Patients were instructed to lie in a dorsal position with the right arm in abduction and measurements were performed on the right lobe of the liver, through intercostal spaces [23].

\subsection{Genotyping for SNPS}

Genomic DNA was collected from peripheral blood samples according to standard procedures for genotyping the following genotype variants: PNPLA3 I148M, TM6SF2 E167K, SERPINA1 and
HSD17B13 [24]. Therefore, the 5-nuclease allelic discrimination TaqMan genotyping method was performed by using pre-designed assays from Applied Biosystems (Foster City, CA) according to the manufacturer's instructions on the ViiA7 instrument (Applied Biosystems, Forster City, CA). To verify the received results, $10 \%$ of samples were genotyped in duplicates.

\subsection{Polygenic risk score}

A polygenic risk score was calculated assigning one point for each risk allele. Specifically, PNPLA3 $C / C$ as wild type was counted as 0 points, while PNPLA3 G/C was counted as 1 point and $P N$ PLA3 G/G as 2 points. Patients with TM6SF2 E/E were assigned 0 points, patients with TM6SF2 E/K 1 point and TM6SF2 K/K 2 points. For $H S D 17 B 13$, the $T / T$ variant was counted as 0 points, while patients with HSD17B13 T/TA were deducted -1 point and patients with $H S D 17 B 13 T A / T A$ were deducted -2 points. Finally, patients with SERPINA1 $M / M$ or $M / S$ were assigned 0 points, while patients with SERPINA1 $M / Z$ or $S / Z$ were assigned 1 point and patients with SERPINA1 Z/Z 2 points. This resulted in a polygenic risk score between -2 and 4 points.

\subsection{Statistical analyses}

Statistical analyses were performed using IBM SPSS Statistics 26 (SPSS Inc., Armonk, New York, USA) and GraphPad Prism 8 (GraphPad Software, La Jolla, California, USA). Depending on their distribution, continuous variables were reported as mean \pm standard deviation (SD) or median (IQR). Categorical variables were presented as numbers and proportions of patients. Comparisons of continuous variables were carried out by using Student's $t$-test or Mann-Whitney U test, as applicable. For comparisons of more than two groups, Kruskal-Wallis one-way analysis of variance was used. Proportions of patients were compared using $\chi^{2}$-squared test. Multivariable linear regression analyses were used to assess the associations between SNPs and LSM. Additionally, multivariable logistic regression analyses were used to determine their association with the presence of ACLD. The following covariables were used for regression analyses: Age, body mass index (BMI) and significant alcohol consumption. To assess the influence of risk variants, genotypes were compared binomially as presence of PNPLA3 $\mathrm{G}$-allele (C/C vs. G/C or G/G), TM6SF2 $\mathrm{K}$-allele (E/E vs. E/K or $\mathrm{K} / \mathrm{K}$ ), HSD17B13 TA-allele (T/T vs. T/TA or TA/TA), and SERPINA1 Z-allele ( $\mathrm{M} / \mathrm{M}$ or $\mathrm{M} / \mathrm{S}$ vs. $M / Z$ or $\mathrm{S} / \mathrm{Z}$ or $\mathrm{Z} / \mathrm{Z}$ ). To test whether the allele frequency observed in our study conformed to an ideal population in Hardy-Weinberg equilibrium, we calculated $\chi^{2}$-squared tests as previously described with values of $\geq 3.84$ indicating a significant difference [25]. A two-sided $P$ value $\leq 0.05$ was considered as statistically significant.

\section{Results}

\subsection{Study population and patient characteristics (Fig. 1, Table 1)}

In total, 875 patients were evaluated for inclusion in this study (Fig. 1). Following other exclusion criteria, 779 patients could finally be analyzed. The majority of patients were male ( $n=476$, $61.1 \%)$ with a mean age of $51.7 \pm 15.8$ years. NAFLD was the most common etiology of liver disease $(n=511,65.6 \%)$, followed by ALD ( $n=118,15.1 \%)$, chronic hepatitis $C$ virus $(n=79,10.1 \%)$ and chronic hepatitis B virus disease ( $n=44,5.6 \%)$. Mean BMI was $26.7 \pm 5.0 \mathrm{~kg} / \mathrm{m}^{2}$, median LSM was $6.0 \mathrm{kPa}$ (IQR: 4.6-9.2) and median CAP was $273 \mathrm{~dB} / \mathrm{m}$ (IQR: 223-321). 171 (22.0\%), 102 (13.1\%) and $77(9.9 \%)$ patients had $\mathrm{LSM} \geq 10.0, \geq 15.0$ and $\geq 20.0 \mathrm{kPa}$. Overall, ACLD was present in 217 (27.9\%) patients. 


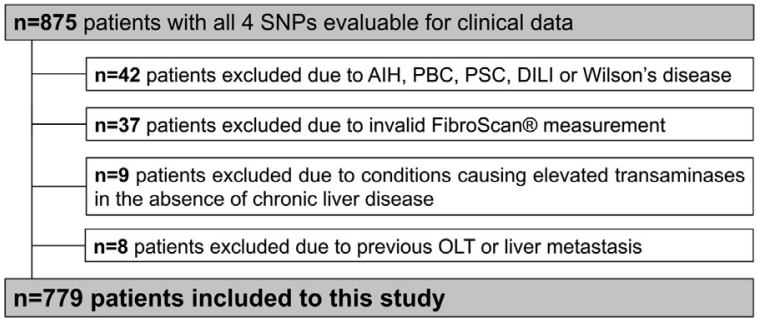

Fig. 1. Study flowchart.

Abbreviations: ACLD advanced chronic liver disease AIH autoimmune hepatitis DILI drug-induced liver disease HCC hepatocellular carcinoma OLT orthotopic liver transplantation PBC primary biliary cholangitis PSC primary sclerosing cholangitis SNP single nucleotide polymorphism TIPS transjugular intrahepatic portosystemic shunt.

\subsection{Prevalence of risk alleles (Table 1)}

Overall, the PNPLA3 wild-type $(C / C)$ was present in 394 patients (50.6\%), whereas the heterozygous $(G / C)$ and the homozygous mutant-type $(G / G)$ were present in 303 (38.9\%) and $82(10.5 \%)$ patients. Thus, PNPLA3 rs738409 G-allele frequency was 0.2997, and $\chi^{2}$ was 4.2027 when compared to an ideal population in HardyWeinberg equilibrium. The TM6SF2 E/E genotype was present in 641 patients $(82.3 \%)$, while 133 patients $(17.1 \%)$ harbored the heterozygous allele (TM6SF2 $E / K$ ) and 5 patients $(0.6 \%)$ the homozygous K-allele (TM6SF2 K/K). TM6SF2 rs58542926 K-allele frequency was 0.0918 and $\chi^{2}$ was 0.4511. Furthermore, HSD17B13 wild-type was found in $59.2 \%(n=461)$, the heterozygous allele HSD17B13 T/TA in $33.9 \%(n=264)$ and the homozygous TA-allele in $6.9 \%$ $(n=54)$ patients $\left(\chi^{2}=3.5731\right)$. For SERPINA1, the allele distribution was as follows: 707 with $M / M(90.8 \%), 26$ with $M / S$ (3.3\%), 44 with $M / Z(5.6 \%)$ and 2 with $Z / Z(0.3 \%)$.

\subsection{Association with liver disease (Table 2, Supplementary Tables} 1-4)

First, we compared LSM and CAP values as well as transaminase levels across patients with different genotypes of the respective single SNPs (univariable analyses). Differences were only observed across PNPLA3 genotypes for LSM, AST and ALT (Supplementary Table 1). For all other genotypes, no differences in these parameters were observed following univariable analyses. Similarly, the prevalence of HCC, ACLD and type-2 diabetes mellitus (T2DM) only significantly differed among PNPLA3 genotypes while no differences existed for the others (Supplementary Table 2).

Next, logistic and linear regressions were calculated to assess the impact of the individual risk alleles on disease severity in the presence of age, BMI and alcohol consumption (Table 2A, multivariable analyses). PNPLA3 G-allele was independently associated with higher LSM (adjusted B: 2.508 [95\%CI: 0.887-3.140], $P=0.002$ ) and higher odds of ACLD (aOR: 1.562 [95\%CI: $1.097-2.226$ ], $P=0.013)$. TM6SF2 $\mathrm{K}$-allele did not increase LSM $(P=0.208)$ or the risk of ACLD $(P=0.503)$. HSD17B13 risk allele expression showed a numerical trend in the overall cohort but was neither associated with LSM $(P=0.067)$ nor with presence of ACLD $(P=0.462)$. As for SERPINA1, LSM was significantly associated with presence of $Z$-allele (adjusted B: 4.558 [95\%CI: $1.182-$ 7.934], $P=0.008$ ), whereas it was not associated with ACLD (aOR: 1.858 [95\%Cl: 0.939-3.678], $P=0.075$ ).

Next, we aimed to assess the influence of each risk allele in the presence/absence of other risk alleles (Table 2B). PNPLA3 G-allele remained independently associated with LSM (adjusted B: 2.409 [95\%Cl: $0.793-4.026], P=0.004$ ) or ACLD (aOR: 1.568 [95\%Cl: 1.098-2.238], $P=0.013$ ). However, only SERPINA1 Z-allele was independently associated with LSM (adjusted B: 4.620 [95\%CI: 1.2607.979], $P=0.007$ ) while the other risk alleles were neither signif- icantly associated with LSM nor ACLD after correcting for PNPLA3. These associations were similar when including T2DM and lowdensity lipoprotein (Supplementary Table 3) or additionally adding gender (Supplementary Table 4) as covariables.

\subsection{Polygenic risk score (Table 3)}

Finally, we created a polygenic risk score to investigate whether a simple coding system for risk alleles might increase significance of risk stratification. Overall, -2 points, were allocated to $17 \mathrm{pa}-$ tients (2.3\%) and -1 points were allocated 131 patients (16.8\%). The majority of patients had 0 points $(n=301,38.6 \%)$. Moreover, 1 point was observed in $28.6 \%(n=223), 2$ points in $12.1 \%$ $(n=94)$ and 3 points in $1.5 \%(n=12)$ patients. The maximum score of 4 points was seen in one patient $(0.1 \%)$. Notably, this score was significantly associated with LSM (adjusted B: $0.948[95 \% \mathrm{Cl}$ : 0.153-1.743], $P=0.020)$. However, it was not predictive for ACLD $(P=0.177)$.

\section{Discussion}

In this study, we aimed at investigating the usefulness of genotyping of four important SNPs (PNPLA3, TM6SF2, HSD17B13, SERPINA1) in clinical routine in the assessment of the severity of chronic liver disease. Therefore, we analysed a cohort of 779 consecutive patients with symptomatic and/or suspected liver disease at the time of their first contact with a hepatologist at a tertiary care center. We confirmed the established association of PNPLA3 with disease severity but identified SERPINA1 as another central disease modifying gene in liver disease patients.

At first, we identified a different distribution of PNPLA3 variants in our cohort when compared to an ideal population in HardyWeinberg-equilibrium while their distribution was not different for HSD17B13, TM6SF2, and SERPINA1. Furthermore, the prevalence of PNPLA3 risk allele in our cohort was higher than reported in literature while it was similar for TM6SF2, HSD17B13 and SERPINA1 [14,26].

These data suggest that PNPLA3 risk variants seem to be enriched in patients getting specialist referral for the assessment of suspected liver disease while no such indication was observed for other genotypes. Thus, the effect of PNPLA3 risk variant carriage may translate into a higher risk of developing clinically overt liver disease. Although this was not observed for HSD17B13, TM6SF2, and SERPINA1, these variants are significantly less common in the general population which might attenuate their influence on disease severity on a population level despite a strong biological effect and pathophysiological influence in the individual patient. Given the well-established role of TM6SF2 and HSD17B13 variants in liver disease, the insignificant associations for these variants in our study is likely due to a lack of statistical power. The association of $P N-$ PLA3 with liver disease at our unselected cohort goes in line with data from the population based National Health and Nutrition Examination Survey (NHANES) study demonstrating increased mortality from liver disease in carriers of PNPLA3 risk variants [27]. In particular, PNPLA3 risk variants were associated with LSM and the clinical diagnosis of ACLD in our cohort while no such link was found for the other genotypes. Again, this observation contributes to the increasing knowledge that the PNPLA3 risk allele might indeed be the central genetic risk factor for developing symptomatic liver disease [28]. Although not from unselected consecutive patients, previous data have demonstrated that PNPLA3 variants are a strong risk factor for development of liver cirrhosis and HCC $[4,29,30]$. Thus, genotyping for PNPLA3 may provide information beyond the assessment of liver disease severity at the time of assessment (i.e., when liver disease starts to be clinically evident). Therefore, it might impact follow-up strategies such as intervals of 
Table 1

Patient and disease characteristics.

Abbreviations: CAP controlled attenuation parameter IQR interquartile range SD standard deviation T2DM type 2 diabetes mellitus.

\begin{tabular}{|c|c|c|c|c|}
\hline Characteristics & \multicolumn{4}{|l|}{ Overall $n=779$} \\
\hline \multicolumn{5}{|l|}{ Clinical data } \\
\hline Age, $y$, mean $\pm S D$ & \multicolumn{4}{|l|}{$51.7 \pm 15.8$} \\
\hline Male sex, $\mathrm{n}(\%)$ & \multicolumn{4}{|l|}{$n=476(61.1 \%)$} \\
\hline BMI, $\mathrm{kg} / \mathrm{m}^{2}$, mean $\pm \mathrm{SD}$ & \multicolumn{4}{|l|}{$26.7 \pm 5.0$} \\
\hline LSM, kPa, median (IQR) & \multicolumn{4}{|l|}{$6.0(4.6-9.2)$} \\
\hline $\mathrm{LSM} \geq 10 \mathrm{kPa}, \mathrm{n}(\%)$ & \multicolumn{4}{|l|}{$n=171(22.0 \%)$} \\
\hline $\mathrm{LSM} \geq 15 \mathrm{kPa}, \mathrm{n}(\%)$ & \multicolumn{4}{|l|}{$n=102(13.1 \%)$} \\
\hline $\mathrm{LSM} \geq 20 \mathrm{kPa}, \mathrm{n}(\%)$ & \multicolumn{4}{|c|}{$n=77(9.9 \%)$} \\
\hline CAP, $\mathrm{dB}$, median (IQR) & \multicolumn{4}{|c|}{$273.0(223.0-321.0)$} \\
\hline \multicolumn{5}{|l|}{ Etiology of liver disease, $n(\%)$} \\
\hline Non-alcoholic fatty liver disease (NAFLD) & \multicolumn{4}{|l|}{$n=511(65.6 \%)$} \\
\hline Alcoholic liver disease (ALD) & \multicolumn{4}{|l|}{$n=118(15.1 \%)$} \\
\hline Hepatitis $\mathrm{C}$ virus infection (HCV) & \multicolumn{4}{|l|}{$n=79(10.1 \%)$} \\
\hline Hepatitis B virus infection (HBV) & \multicolumn{4}{|l|}{$n=44(5.6 \%)$} \\
\hline Unknown liver disease & \multicolumn{4}{|l|}{$n=27(3.5 \%)$} \\
\hline ACLD & \multicolumn{4}{|l|}{$n=217(27.9 \%)$} \\
\hline $\mathrm{HCC}$ & \multicolumn{4}{|l|}{$n=14(1.8 \%)$} \\
\hline T2DM & \multicolumn{4}{|l|}{$n=88(11.3 \%)$} \\
\hline Genotypes, $n(\%)$ & & $p$ & $q$ & $\chi^{2}$ \\
\hline PNPLA3 C/C & $n=394(50.6 \%)$ & 0.700 & 0.300 & 4.2027 \\
\hline PNPLA3 G/C & $n=303(38.9 \%)$ & & & \\
\hline PNPLA3 G/G & $n=82(10.5 \%)$ & & & \\
\hline SERPINA1 M/M & $n=707(90.8 \%)$ & 0.969 & 0.031 & 2.2879 \\
\hline SERPINA1 $\mathrm{M} / \mathrm{S}$ & $n=26(3.3 \%)$ & & & \\
\hline SERPINA1 $M / Z$ & $n=44(5.6 \%)$ & & & \\
\hline SERPINA1 Z/Z & $n=2(0.3 \%)$ & & & \\
\hline TM6SF2 E/E & $n=641(82.3 \%)$ & 0.908 & 0.092 & 0.4511 \\
\hline TM6SF2 E/K & $n=133(17.1 \%)$ & & & \\
\hline TM6SF2 K/K & $n=5(0.6 \%)$ & & & \\
\hline HSD17B13 T/T & $n=461(59.2 \%)$ & 0.761 & 0.239 & 3.5731 \\
\hline HSD17B13 T/TA & $n=264(33.9 \%)$ & & & \\
\hline HSD17B13 TA/TA & $n=54(6.9 \%)$ & & & \\
\hline
\end{tabular}

Table 2

(A) Linear regression analyses (liver stiffness measurement, LSM) and logistic regression analyses (advanced chronic liver disease, ACLD) investigating individual associations of risk alleles with LSM and the presence of ACLD (multivariable analysis). (B) All genetic loci in a combined linear and logistic regression analysis.

Covariables for both approaches were: Age (yrs), BMI $\left(\mathrm{kg} / \mathrm{m}^{2}\right)$, significant alcohol abuse (women $\geq 2$ drinks per day, men $\geq 3$ drinks per day).

Abbreviations: ACLD advanced chronic liver disease CAP controlled attenuation parameter C confidence interval IQR interquartile range LSM liver stiffness measurement.

\begin{tabular}{lll}
\hline$A$ & LSM & ACLD \\
\hline PNPLA3 $(G=1)$ & $2.508(0.887-4.130), P=\mathbf{0 . 0 0 2}$ & $1.562(1.097-2.226), P=\mathbf{0 . 0 1 3}$ \\
TM6SF2 $(K=1)$ & $1.361(-0.758-3.481), P=0.208$ & $0.853(0.535-1.359), P=0.503$ \\
HSD17B13 $(T A=1)$ & $1.551(-0.106-3.208), P=0.067$ & $1.144(0.800-1.635), P=0.462$ \\
SERPINA1 $(Z=1)$ & $4.558(1.182-7.934), P=\mathbf{0 . 0 0 8}$ & $1.858(0.939-3.678), P=0.075$ \\
\hline & LSM & ACLD \\
B & $2.409(0.793-4.026), P=\mathbf{0 . 0 0 4}$ & $1.568(1.098-2.238), P=\mathbf{0 . 0 1 3}$ \\
\hline PNPLA3 $(G=1)$ & $1.547(-0.554-3.648), P=0.149$ & $0.851(0.529-1.368), P=0.504$ \\
TM6SF2 $(K=1)$ & $1.344(-0.302-2.990), P=0.109$ & $1.106(0.771-1.587), P=0.584$ \\
HSD17B13 $(T A=1)$ & $4.620(1.260-7.979), P=\mathbf{0 . 0 0 7}$ & $1.841(0.921-3.680), P=0.084$ \\
SERPINA1 $(Z=1)$ & &
\end{tabular}

regular consultations, ultrasound screening for HCC, and contribute to a personalized approach in clinical hepatology.

Although other SNPs are continuously being discovered in genome-wide association studies, their impact on disease severity seems to be weaker compared to PNPLA3 [13]. Since PNPLA3/TM6SF2/HSD17B13 interact with different steps in lipid homeostasis [31], a potentiation of their harmful effect leading to disease progression could be expected. While PNPLA3 promotes intracellular lipid accumulation in the liver by impairing mobilization of various lipid species from lipid droplets and reducing the lipidation of very low-density lipoprotein (VLDL) [32], the TM6SF2 variant is linked to inhibition of VLDL secretion from hepatocytes [33]. Although the exact function of HSD17B13 remains unknown, this protein also increases size and accumulation of lipid droplets in hepatocytes [34] while the loss-of-function variant in HSD17B13 seems to counteract this mechanism. Finally, SERPINA1 acts differently by augmenting proteotoxicity (i.e., accumulation of alpha-1antitrypsin) in the endoplasmic reticulum [35]. To test whether the different biological mechanisms of these variants potentuate each other, we combined them into a polygenic risk score. This polygenic risk score was significantly associated with LSM, but not with the presence of ACLD in our study, indicating that carriage of several SNPs might increase an individual's risk for advanced fibrosis. However, the strength of the observed associations of the combined genetic risk score was weaker than that of PNPLA3 alone. This indicates that PNPLA3 alone represents a central SNP to which 


\section{Table 3}

Overview of genetic landscape using a polygenic risk score, and linear and logistic regression analyses investigating the association of polygenic risk score with liver stiffness measurement (LSM) and presence of advanced chronic liver disease (ACLD). Covariables were: Age (yrs), BMI $\left(\mathrm{kg} / \mathrm{m}^{2}\right)$, significant alcohol abuse (women $\geq 2$ drinks per day, men $\geq 3$ drinks per day). Abbreviations: ACLD advanced chronic liver disease CI confidence interval LSM liver stiffness measurement.

\begin{tabular}{llll}
\hline Polygenic risk score & \multicolumn{3}{l}{} \\
\hline Points & $n$ & $\%$ & Cumulative\% \\
\hline-2 & 17 & 2.3 & 2.3 \\
-1 & 131 & 16.8 & 19.0 \\
0 & 301 & 38.6 & 57.6 \\
1 & 223 & 28.6 & 86.3 \\
2 & 94 & 12.1 & 98.3 \\
3 & 12 & 1.5 & 99.9 \\
4 & 1 & 0.1 & 100.0 \\
Overall & 779 & 100.0 & 100.0 \\
Polygenic risk score & 0.948 & $(0.153-1.743) P=\mathbf{0 . 0 2 0}$ & $1.124(0.949-1.332) P=0.177$ \\
\hline
\end{tabular}

Polygenic risk score: PNPLA3 $\rightarrow 0 / 1 / 2 ;$ TM6SF2 $\rightarrow 0 / 1 / 2 ;$ HSD17B13 $\rightarrow 0 /-1 /-2$; SERPINA1 $\rightarrow 0 / 1 / 2$.

the other SNPs only add little information. Although one might argue that these variants should be weighted according to the strength of their association with disease severity, we chose to assign one point for each risk allele to treat them equally. Importantly, a customized weighting would need a meta-analysis of all published studies evaluating the four SNPs used in this study (PNPLA3, TM6SF2, HSD17B13, SERPINA1) together, which has not been done so far. Arbitrarily choosing a coefficient for each risk allele would artificially modify the relevance of the respective risk allele which is - when seen from a natural perspective - either present (1) or not (0). Importantly, several studies have already aimed to identify the utility of polygenic risk scores to predict disease severity in NAFLD [36-38]. Just recently, Bianco and colleagues [39] identified a polygenic risk score combining PNPLA3TM6SF2-GCKR-MBOAT7 as predictive for HCC. However, none has included TM6SF2 and HSD17B13.

Interestingly, SERPINA1 was independently associated with LSM in our cohort. This goes in line with recent findings of Strnad et al. [14] who found that the SERPINA1 Z-allele was associated with disease severity and an increased risk to develop cirrhosis in patients with NAFLD and ALD. This association was independent of PNPLA3, TM6SF2 and MBOAT7, and stronger than other risk variants for the development of NAFLD/ALD cirrhosis [13]. In our study, the regression coefficient regarding the association with LSM was nearly twice as high as the coefficient of PNPLA3, indicating a stronger influence of LSM. Although a numerically higher aOR was also observed for the presence of ACLD, this did not achieve statistical significance, probably due to a significantly lower prevalence of SERPINA1 risk alleles. Therefore, our data support that the SERPINA1 risk allele - even in heterozygous form - provides additional information for risk stratification, which likewise warrants genotyping in patients with liver disease. From a clinical point, our data suggest that at least genotyping for PNPLA3 variants may add additional value in the routine work-up of liver disease patients, while the usefulness of other variants in this setting is not supported by our findings, except for a potential role of the SERPINA1 variant in the progression to advanced stages of fibrosis. The routine use of genotyping is also further limited by the respective financial burden. However, as these are highly variable in different regions, the decision will need to be made according to the weight of the individual factors.

Our study indicates that neither of the investigated variants are linked to CAP which represents a widely used non-invasive estimate for the extent of hepatic steatosis. However, this is likely due to the nature of CAP measurements being variable, unable to grade hepatic steatosis [40] while being influenced by numerous factors including alcohol intake, BMI, T2DM or fibrosis, particularly in our cohort of mixed etiologies [22,41,42]. Therefore, our findings do not argue against the fact that these SNPs promote the development of hepatic steatosis, which has been well-documented by numerous studies.

This study has several limitations. To begin with, it is a crosssectional retrospective study showing only one time point in the course of liver disease. Longitudinal data on liver-related morbidity and mortality are needed to support our findings. However, as genetic markers remain unchanged, the observed association in fact reflects the impact of the variant over the entire observed lifetime. Moreover, since we aimed to assess the utility of genotyping in clinical routine, the cohort being analyzed combines different etiologies of CLD reflecting the heterogeneity of risk factors typical for the general population. Hence, the set environmental risk factors such as diet, physical exercise, amount of alcohol, may be composed differently in any individual included. We aimed to reduce the heterogeneity of the cohort by only including the common fatty liver and viral diseases which are expected to mostly have a relatively continuous chronic course of disease progression in an individual. Although desirable, genotyping for membrane bound $\mathrm{O}$-acyltransferase domain-containing 7 gene (MBOAT7) was not available in our cohort as this was not part of the routine clinical program during the study period.

In conclusion, we provide data on the usefulness of genotyping for important risk alleles in a cohort of CLD patients with Caucasian background in clinical routine. Specifically, we confirm the strong association between PNPLA3 and liver disease severity. Although other SNPs including SERPINA1 might add information on further risk of developing (severe) liver disease, PNPLA3 was identified to be of central importance and its use may be justified in the clinical routine. Combining multiple risk alleles into a polygenic score might increase granularity of genetic risk stratification.

\section{Declaration of Competing Interest}

None of the authors have potential conflicts of interest with regard to this manuscript to disclose. However, some of the authors have conflicts of interest outside the submitted work: C.D. is part of the scientific advisor board of SPAR Österreich WarenhandelsAG.

\section{Funding}

Not applicable. 


\section{Availability of data}

All data generated or analyzed during this study are included in this published article (and its supplementary information files).

\section{Code availability}

Not applicable.

\section{Author contributions}

Study concept and design (L.B., G.S., E.A.), acquisition of data (L.B., G.S., H.O., S.Z., M.S., L.D., D.N., A.F., F.S., C.D., E.A.), analysis and interpretation of data (L.B., G.S., E.A.), drafting of the manuscript (L.B., G.S., E.A.), critical revision of the manuscript for important intellectual content (L.B., G.S., H.O., S.Z., M.S., L.D., D.N., A.F., F.S., C.D., E.A.). The first draft of the manuscript was written by L.B., G.S. and E.A. and all authors commented on previous versions of the manuscript. All authors read and approved the final manuscript.

\section{Ethics approval}

This study was approved by the ethics committee of Salzburg (Ethikkommission für das Bundesland Salzburg; http://www. salzburg.gv.at/ethikkommission) and performed in accordance with the standards of the Declaration of Helsinki. Since it was a retrospective analysis of available clinical data, the requirement of a written informed consent was waived.

\section{Consent for publication}

Not applicable.

\section{Abbreviations}

95\%CI 95\% confidence interval ACLD advanced chronic liver disease aHR adjusted hazard ratio aOR adjusted odds ratio BMI body mass index CACLD compensated advanced chronic liver disease CAP controlled attenuation parameter CLD chronic liver disease CSPH clinically significant portal hypertension CPS Child-TurcottePugh-Score HCC hepatocellular carcinoma HE hepatic encephalopathy HR hazard ratio HSD17B13 $17 \beta$-hydroxysteroid dehydrogenase type $13 \mathrm{IQR}$ interquartile range LSM liver stiffness measurement LT liver transplantation MELD model for end-stage liver disease NAFLD non-alcoholic fatty liver disease NASH non-alcoholic steatohepatitis PNPLA3 patatin-like phospholipase domain-containing protein 3 SD standard deviation SERPINA1 serpin family A member 1 TM6SF2 transmembrane 6 superfamily 2 T2DM type- 2 diabetes mellitus

\section{Supplementary materials}

Supplementary material associated with this article can be found, in the online version, at doi:10.1016/j.dld.2021.06.015.

\section{References}

[1] Sookoian S, Pirola CJ. Meta-analysis of the influence of I148M variant of patatin-like phospholipase domain containing 3 gene (PNPLA3) on the susceptibility and histological severity of nonalcoholic fatty liver disease. Hepatology 2011;53:1883-94.

[2] Romeo S, Kozlitina J, Xing C, et al. Genetic variation in PNPLA3 confers susceptibility to nonalcoholic fatty liver disease. Nat Genet 2008;40:1461-5.

[3] Buch S, Stickel F, Trépo E, et al. A genome-wide association study confirms PNPLA3 and identifies TM6SF2 and MBOAT7 as risk loci for alcohol-related cirrhosis. Nat Genet 2015;47:1443-8.
[4] Salameh H, Raff E, Erwin A, et al. PNPLA3 gene polymorphism is associated with predisposition to and severity of alcoholic liver disease. Am J Gastroenterol 2015:110:846-56.

[5] Stickel F, Buch S, Lau K, et al. Genetic variation in the PNPLA3 gene is associated with alcoholic liver injury in caucasians. Hepatology 2011;53:86-95.

[6] Trépo E, Gustot T, Degré D, et al. Common polymorphism in the PNPLA3/adiponutrin gene confers higher risk of cirrhosis and liver damage in alcoholic liver disease. J Hepatol 2011;55:906-12.

[7] Atkinson SR, Way MJ, McQuillin A, et al. Homozygosity for rs738409:g in PNPLA3 is associated with increased mortality following an episode of severe alcoholic hepatitis. J Hepatol 2017;67:120-7.

[8] Stättermayer AF, Scherzer T, Beinhardt S, et al. Review article: genetic factors that modify the outcome of viral hepatitis. Aliment Pharmacol Ther 2014:39:1059-70.

[9] Dongiovanni P, Romeo S, Valenti L. Genetic factors in the pathogenesis of nonalcoholic fatty liver and steatohepatitis. Biomed Res Int 2015;2015:460190.

[10] Unalp-Arida A, Ruhl CE. Patatin-like phospholipase domain-containing protein 3 I148M and liver fat and fibrosis scores predict liver disease mortality in the U.S. population. Hepatology 2020;71:820-34.

[11] Kozlitina J, Smagris E, Stender S, et al. Exome-wide association study identifies a TM6SF2 variant that confers susceptibility to nonalcoholic fatty liver disease. Nat Genet 2014;46:352-6.

[12] About F, Abel L, Cobat A. HCV-associated liver fibrosis and HSD17B13. N Engl J Med 2018;379:1875-6.

[13] Abul-Husn NS, Cheng X, Li AH, et al. A protein-truncating HSD17B13 variant and protection from chronic liver disease. N Engl J Med 2018;378:1096-106.

[14] Strnad P, Buch S, Hamesch K, et al. Heterozygous carriage of the alpha1-antitrypsin $\mathrm{Pi}^{*} \mathrm{Z}$ variant increases the risk to develop liver cirrhosis. Gut 2019;68:1099-107.

[15] Singal AG, Manjunath H, Yopp AC, et al. The effect of PNPLA3 on fibrosis progression and development of hepatocellular carcinoma: a meta-analysis. Am J Gastroenterol 2014;109:325-34.

[16] Semmler G, Balcar L, Oberkofler H, et al. PNPLA3 and SERPINA1 variants are associated with severity of fatty liver disease at first referral to a tertiary center. J Pers Med 2021:11.

[17] Stickel F, Lutz P, Buch S, et al. Genetic variation in HSD17B13 reduces the risk of developing cirrhosis and hepatocellular carcinoma in alcohol misusers. Hepatology 2020;72:88-102 (Baltimore, Md).

[18] Liu YL, Reeves HL, Burt AD, et al. TM6SF2 rs58542926 influences hepatic fibrosis progression in patients with non-alcoholic fatty liver disease. Nat Commun 2014;5:4309.

[19] EASL-EASD-EASO clinical practice guidelines for the management of non-alcoholic fatty liver disease. J Hepatol 2016;64:1388-402.

[20] Pons M, Rodríguez-Tajes S, Esteban JI, et al. Non-invasive prediction of liver-related events in patients with HCV-associated compensated advanced chronic liver disease after oral antivirals. J Hepatol 2020;72:472-80.

[21] de Franchis R. Expanding consensus in portal hypertension: report of the Baveno VI Consensus Workshop: stratifying risk and individualizing care for portal hypertension. J Hepatol 2015;63:743-52.

[22] Karlas T, Petroff D, Sasso M, et al. Individual patient data meta-analysis of controlled attenuation parameter (CAP) technology for assessing steatosis. J Hepatol 2017;66:1022-30.

[23] Semmler G, Wöran K, Scheiner B, et al. Novel reliability criteria for controlled attenuation parameter assessments for non-invasive evaluation of hepatic steatosis. United Eur Gastroenterol J 2020;8:321-31.

[24] Kedenko L, Lamina C, Kedenko I, et al. Genetic polymorphisms at SIRT1 and FOXO1 are associated with carotid atherosclerosis in the SAPHIR cohort. BMC Med Genet 2014;15:112.

[25] Rodriguez S, Gaunt TR, Day IN. Hardy-Weinberg equilibrium testing of biological ascertainment for mendelian randomization studies. Am J Epidemiol 2009;169:505-14.

[26] Kozlitina J. Genetic risk factors and disease modifiers of nonalcoholic steatohepatitis. Gastroenterol Clin N Am 2020;49:25-44.

[27] Wijarnpreecha K, Scribani M, Raymond P, et al. PNPLA3 gene polymorphism and liver- and extrahepatic cancer-related mortality in the United States. Clin Gastroenterol Hepatol 2021;May 19(5):1064-6 Epub 2020 Apr 29. doi:10.1016/ j.cgh.2020.04.058.

[28] Krawczyk M, Liebe R, Lammert F. Toward genetic prediction of nonalcoholic fatty liver disease trajectories: PNPLA3 and beyond. Gastroenterology 2020;158:1865-80 e1.

[29] Hassan MM, Kaseb A, Etzel CJ, et al. Genetic variation in the PNPLA3 gene and hepatocellular carcinoma in USA: risk and prognosis prediction. Mol Carcinog 2013:52(Suppl 1):E139-47.

[30] Trépo E, Romeo S, Zucman-Rossi J, et al. PNPLA3 gene in liver diseases. J Hepatol 2016;65:399-412.

[31] Taliento AE, Dallio M, Federico A, et al. Novel insights into the genetic landscape of nonalcoholic fatty liver disease. Int J Environ Res Public Health 2019;16:2755.

[32] Pirazzi C, Adiels M, Burza MA, et al. Patatin-like phospholipase domain-containing 3 (PNPLA3) I148M (rs738409) affects hepatic VLDL secretion in humans and in vitro. J Hepatol 2012;57:1276-82.

[33] Zhou Y, Llauradó G, Orešič M, et al. Circulating triacylglycerol signatures and insulin sensitivity in NAFLD associated with the E167K variant in TM6SF2. Hepatol 2015;62:657-63.

[34] Su W, Wang Y, Jia X, et al. Comparative proteomic study reveals $17 \beta$-HSD13 
as a pathogenic protein in nonalcoholic fatty liver disease. Proc Natl Acad Sci U S A 2014;111:11437-42.

[35] Teckman JH, An JK, Blomenkamp K, et al. Mitochondrial autophagy and injury in the liver in alpha 1-antitrypsin deficiency. Am J Physiol Gastrointest Liver Physiol 2004;286:G851-62.

[36] León-Mimila P, Vega-Badillo J, Gutiérrez-Vidal R, et al. A genetic risk score is associated with hepatic triglyceride content and non-alcoholic steatohepatitis in Mexicans with morbid obesity. Exp Mol Pathol 2015;98:178183.

[37] Kawaguchi T, Shima T, Mizuno M, et al. Risk estimation model for nonalcoholic fatty liver disease in the Japanese using multiple genetic markers. PLoS ONE 2018;13:e0185490.

[38] Dongiovanni P, Stender S, Pietrelli A, et al. Causal relationship of hepatic fat with liver damage and insulin resistance in nonalcoholic fatty liver. J Intern Med 2018;283:356-70.
[39] Bianco C, Jamialahmadi O, Pelusi S, et al. Non-invasive stratification of hepatocellular carcinoma risk in non-alcoholic fatty liver using polygenic risk scores. J Hepatol. Apr 2021;74(4):775-82 Epub 2020 Nov 25. doi:10.1016/j.jhep.2020. 11.024

[40] Runge JH, Smits LP, Verheij J, et al. MR spectroscopy-derived proton density fat fraction is superior to controlled attenuation parameter for detecting and grading hepatic steatosis. Radiology 2018;286:547-56

[41] Semmler G, Stift J, Scheiner B, et al. Performance of controlled attenuation parameter in patients with advanced chronic liver disease and portal hypertension. Dig Dis Sci 2019;64:3642-51.

[42] Petta S, Wong VW, Camma C, et al. Improved noninvasive prediction of liver fibrosis by liver stiffness measurement in patients with nonalcoholic fatty liver disease accounting for controlled attenuation parameter values. Hepatology 2017;65:1145-55 (Baltimore, Md). 